\title{
Emergency lower gastrointestinal endoscopy performed safely in a COVID-19 patient on extracorporeal membrane oxygenation (ECMO) with hemorrhagic shock
}

\author{
Yoshitsugu Misumi $^{1} \cdot$ Yuko Fujii $^{1} \cdot$ Arino Yaguchi $^{2} \cdot$ Kouichi Nonaka $^{1}$
}

Received: 29 March 2021 / Accepted: 8 May 2021 / Published online: 18 May 2021

(c) Japanese Society of Gastroenterology 2021

\begin{abstract}
Emergency endoscopy in coronavirus disease 2019 (COVID-19) patients should be avoided whenever possible to ensure the safety of medical staff; however, it may be unavoidable in some cases. We report a case of emergency lower gastrointestinal endoscopy performed with full personal protective equipment in a patient on extracorporeal membrane oxygenation with severe COVID-19 pneumonia admitted in a restricted area under negative pressure in the intensive care unit. To avoid the risk of fecal-oral transmission of the severe acute respiratory syndrome coronavirus 2 (SARS-CoV-2) during the procedure, the patient's lower body was covered with a $2 \mathrm{~m}^{2}$ vinyl sheet with an aperture (diameter, approximately $2 \mathrm{~cm}$ ). None of the medical staff involved exhibited any signs of SARS-CoV-2 infection after the procedure. Although patients with severe COVID-19 pneumonia on extracorporeal membrane oxygenation have a high risk of bleeding, we believe that emergency lower endoscopy can be safely performed in such patients by reducing exposure to dispersed feces and using full personal protective equipment.
\end{abstract}

Keywords COVID-19 · Infection control · Lower Gastrointestinal Endoscopy · VV-ECMO

\section{Introduction}

The severe acute respiratory syndrome coronavirus 2 (SARS-CoV-2) has spread rapidly [1] and has greatly affected endoscopic medical care. Regarding emergency endoscopy in SARS-CoV-2-positive patients, non-urgent procedures should be avoided due to the risk of infection of healthcare professionals, including endoscopists [2]. However, as the infection spreads, an emergency upper endoscopy for upper gastrointestinal bleeding that typically affects hemodynamic stability is unavoidable. There are few reports of endoscopy in SARS-CoV-2-positive patients [3]. Upper gastrointestinal endoscopy is a procedure with a high risk of aerosol generation, which is one

Yoshitsugu Misumi yoshikft34@gmail.com

1 Department of Digestive Endoscopy, Tokyo Women's Medical University, 8-1, Kawada-cho, Shinjuku-ku, Tokyo 162-8666, Japan

2 Department of Critical Care and Emergency Medicine, Tokyo Women's Medical University, 8-1, Kawada-cho, Shinjuku-ku, Tokyo 162-8666, Japan of the routes of SARS-CoV-2 infection. Various infection prevention measures during endoscopy have been reported [4]. Lower gastrointestinal bleeding can often be treated conservatively; however, an emergency lower endoscopy is unavoidable if the bleeding affects hemodynamic stability. There are few reports of cases in which emergency lower endoscopy has been safely performed in patients infected with SARS-CoV-2 (coronavirus disease 2019 [COVID-19] patients). Similar to aerosols, the fecal-oral route has been considered a potential route of SARS-CoV-2 infection [5]. Therefore, infection control measures are warranted when performing lower gastrointestinal endoscopy in COVID-19 patients. We previously reported a case wherein emergency upper gastrointestinal endoscopy was safely performed in a COVID-19 patient [6]. Here, we report a case in which lower gastrointestinal endoscopy was safely performed in a patient with severe COVID-19 pneumonia on extracorporeal membrane oxygenation (ECMO) by adopting simple droplet precautions. 


\section{Case report}

A 70-year-old man consulted a local physician with complaints of fever above $38^{\circ} \mathrm{C}$ and persistent cough for 4 days. Oropharyngeal swabs collected on the same day were positive for SARS-CoV-2 based on a reverse transcriptase-polymerase chain reaction assay. A computed tomography (CT) scan revealed infiltration shadows in both lungs, and the patient was diagnosed with COVID-19 pneumonia (Fig. 1). Considering concurrent respiratory failure, the patient was transferred to our negative pressure isolation intensive care unit (ICU), and medical treatment was initiated. Antibiotics, dexamethasone, and remdesivir were initiated for pneumonia, and heparin (10,000 IU) was administered to prevent thrombosis. Due to an exacerbation of the respiratory failure, the patient was intubated on day 2 . As there was no improvement in respiratory status, veno-venous ECMO (VV-ECMO) was introduced on day 11 , and the patient required strict anticoagulant therapy. In addition, since renal function gradually worsened, continuous hemodiafiltration was introduced on day 11, alongside treatment with nafamostat mesylate. On day 12 , the patient was referred to our department for consultation after he passed a large amount of bloody stools. A CT scan conducted at the time of admission showed multiple colonic diverticula in the sigmoid colon, suggesting diverticular bleeding. Conservative treatment was also considered; however, spontaneous hemostasis could not be achieved in the patient due to anticoagulant therapy, and the patient also exhibited hemorrhagic shock. Therefore, an emergency lower gastrointestinal endoscopy was deemed necessary. During blood sampling before endoscopy, the following values were observed: hemoglobin $8.5 \mathrm{~g} / \mathrm{dl}$, PT

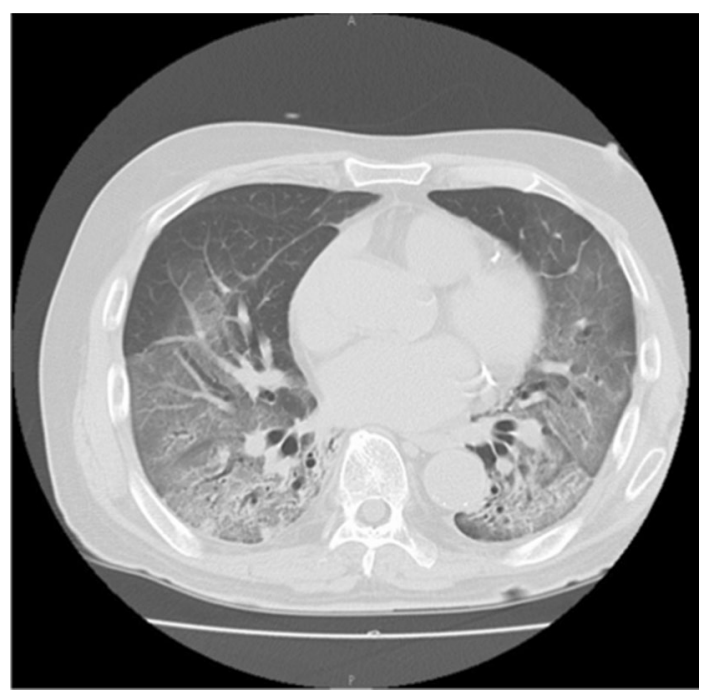

Fig. 1 Computed tomography images showing infiltration shadows in both lungs
$75.1 \%$, activated partial thromboplastin time $51.1 \mathrm{~s}$, fibrin/ fibrinogen degradation products $91.6 \mu \mathrm{g} / \mathrm{ml}$, D-dimer 40.4 $\mu \mu \mathrm{g} / \mathrm{ml}$, platelets $52,000 / \mu \mathrm{l}$, prothrombin time $13.5 \mathrm{~s}$. Vital signs were pulse $96 \mathrm{bpm}$, body temperature $37.0^{\circ} \mathrm{C}$, blood pressure $76 / 57 \mathrm{mmHg}$, respiratory rate 12 breaths/minute. After receiving erythrocyte and platelet transfusions, endoscopy was performed under sedation by two skilled endoscopists in the SARS-CoV-2-positive zone in the ICU after informed consent was obtained. In addition, due to the overall condition of the patient, the lower gastrointestinal endoscopy was performed without pre-treatment. As recommended by various gastroenterology societies [7], the procedure was performed with full personal protection equipment (PPE), including electric-fan facemasks with $99.7 \%$ filtration capability, double gloves, a hairnet, a water-resistant disposable gown, and shoe covers (Fig. 2). The light source and endoscope used were exclusively for the SARS-CoV2-positive zone. To prevent fecal-oral transmission, a $2 \mathrm{~m}^{2}$ vinyl sheet with an aperture of about $2 \mathrm{~cm}$ in diameter was used to cover the entire lower body of the patient. The scope was inserted through the aperture in the vinyl sheet; hence, no stool dispersed out of the vinyl sheet (Fig. 3a, b). Since there was no pre-treatment and insertion was difficult, the

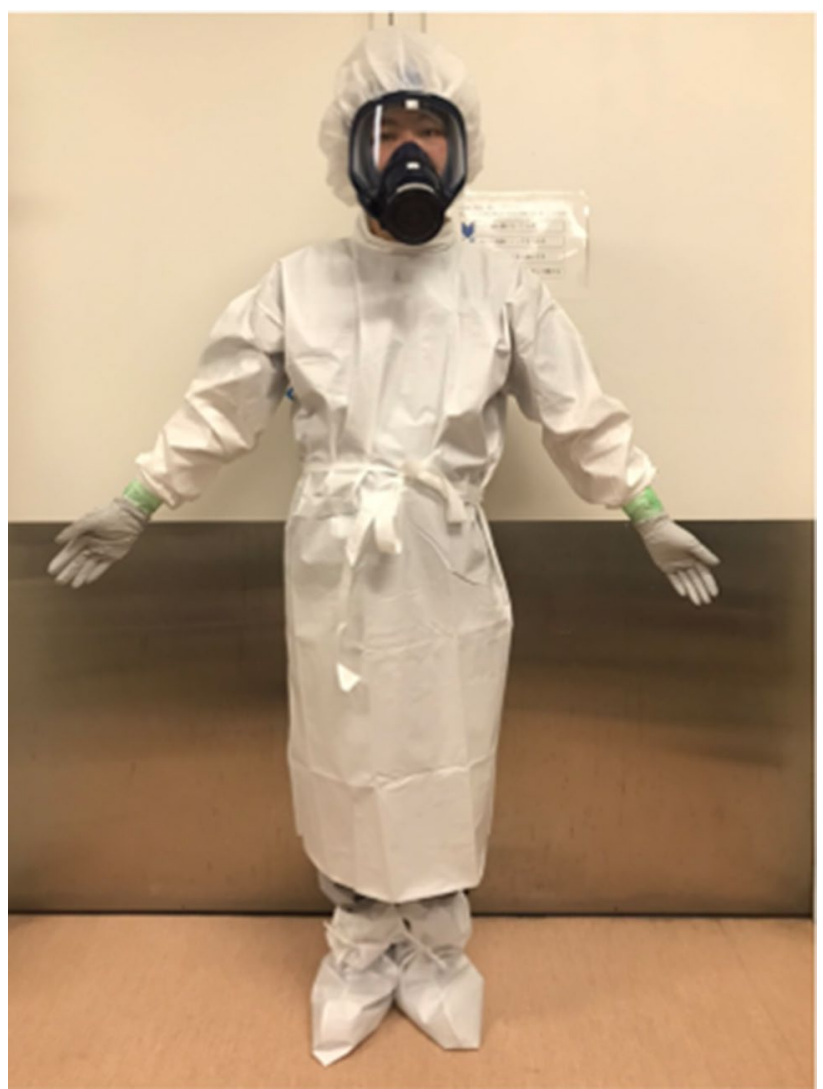

Fig. 2 Actual full personal protective equipment (respirator, double gloves, hairnet, water-resistant disposable gown, shoe covers, and face shield/goggles) 
Fig. 3 a A $2 \mathrm{~m}^{2}$ vinyl sheet with an aperture in the center, (b) The lower half of the body was covered with a vinyl sheet to avoid the dispersion of stool
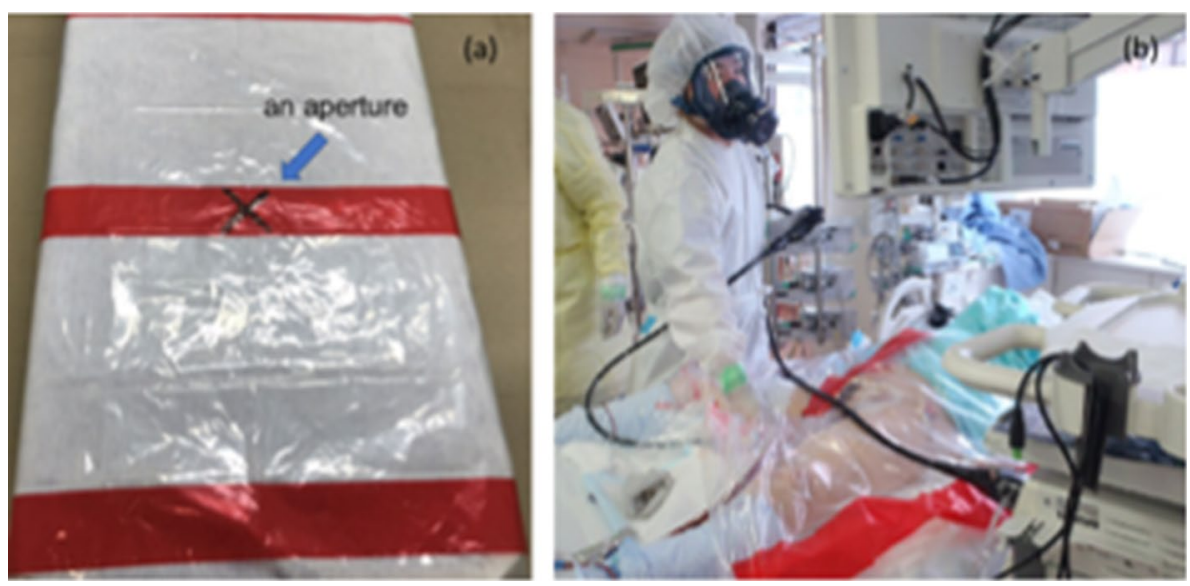

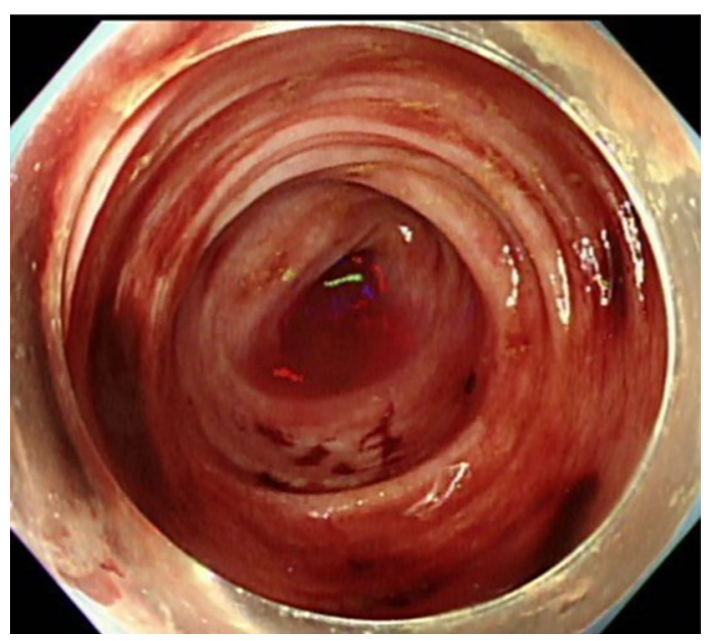

Fig. 4 Bloody stools were noted; however, the source of bleeding was unclear

progression of the scope was limited to the ascending colon. The stool was washed away as much as possible during the procedure. Since fresh blood was observed near the sigmoid colon and multiple diverticula were observed in the sigmoid colon, the procedure was conducted very carefully. However, there was no clear source of bleeding (Fig. 4). After the procedure, we strictly adhered to infection zones in the ICU, and when we took off the PPE, we took great care not to touch the non-sterile side. After carefully wiping the scope and light source with an $80 \%$ ethanol sheet and a $0.5 \%$ accelerated hydrogen peroxide sheet, the surface of the scope was cleaned in the ICU washroom by staff equipped with full PPE, in accordance with routine endoscope cleaning/sterilizing procedures mandated by the Japan Gastroenterological Endoscopy Technicians Society. Subsequently, the forceps channel was washed several times under running water and disinfected with $0.3 \%$ peracetic acid. The accessory equipment used for the procedure were discarded in the SARSCoV-2-positive zone.
Subsequently, heparin administration was discontinued for 2 days to prevent re-bleeding, and since no re-bleeding was observed, the same dose of heparin was resumed from day 14 . The respiratory status of the patient remained stable, and treatment for pneumonia including ECMO was ongoing after the endoscopy. The medical staff involved in the endoscopy procedure were followed up for 2 weeks, and they showed no symptom suggestive of COVID-19.

\section{Discussion}

Unnecessary and non-urgent endoscopy in COVID-19 patients should be avoided as much as possible. However, endoscopy may be unavoidable in cases of upper gastrointestinal bleeding or hemorrhagic shock. A previous report suggested that emergency endoscopy is not necessary for lower gastrointestinal bleeding in COVID-19 patients [8]. However, we encountered a case in which hemorrhagic shock had occurred, and emergency endoscopy was essential.

COVID-19 is known to cause thrombosis, and anticoagulant therapy with heparin is often administered [9]. In particular, COVID-19 patients with severe lung inflammation on ECMO, such as our patient, require strict anticoagulant therapy. ECMO causes acquired von Willebrand syndrome in severely ill COVID-19 patients [10], and in the future, the number of situations in which emergency endoscopy is unavoidable may increase. Endoscopy for COVID-19 patients has been proposed by various academic societies to be conducted in a negative pressure room [7]. In the present case, endoscopy was performed under negative pressure and in a restrictive environment. Furthermore, emergency lower endoscopy could be safely carried out for our COVID-19 patient by combining measures to prevent fecal-oral transmission of SARS-CoV-2, in a situation where the risk of fecal dispersion was slightly high, given that the endoscopy had to be performed without pre-treatment, using full PPE. 
Acknowledgements We would like to thank Editage (www.editage. com) for English language editing.

Authors' contributions YM was involved in the preparation and submission of this manuscript. YM and YF were involved in the emergency endoscopy. $\mathrm{KN}$ was involved in the final correction and proofreading of the manuscript. AY was involved in infection control in the intensive care unit of Tokyo Women's Medical University.

Funding No funding was received for this study.

\section{Compliance with ethical standards}

Conflict of interest All authors declare no conflict of interests.

Ethical statement All procedures followed were performed in accordance with the ethical standards of the 1964 Declaration of Helsinki and its later amendments.

Informed consent Informed consent was obtained from all patients for being included in the study

\section{References}

1. Wu F, Zhao S, Yu B, et al. Author correction: a new coronavirus associated with human respiratory disease in China. Nature. 2020;580:E7.

2. Teng M, Tang SY, Koh CJ. Endoscopy during COVID-19 pandemic: an overview of infection control measures and practical application. World J Gastrointest Endosc. 2020;12:256-65.
3. El Kassas M, Al Shafie A, Abdel Hameed AS, et al. Emergency endoscopic variceal band ligation in a COVID-19 patient presented with hematemesis while on mechanical ventilation. Dig Endosc. 2020;32:812-5.

4. Kikuchi D, Suzuki Y, Hoteya S. Shielding method for the endoscopic procedures during the COVID-19 pandemic. Dig Endosc. 2020;32:e160-1.

5. Heller L, Mota CR, Greco DB. COVID-19 faecal-oral transmission: are we asking the right questions? Sci Total Environ. 2020;729:138919.

6. Misumi Y, Nitta Y, Nonaka K, et al. Emergency upper gastrointestinal endoscopy performed safely in a patient with COVID-19 with suspected hemorrhagic shock. Dig Endosc Open. 2021;3:e2.

7. Dhar J, Samanta J, Kochhar R. Corona virus disease-19 pandemic: the gastroenterologists' perspective. Indian J Gastroenterol 2020;39:220-31.

8. Holzwanger EA, Bilal M, Stallwood CG, et al. Acute lower gastrointestinal bleeding during the COVID-19 pandemic - less is more! Endoscopy. 2020;52:816-7.

9. Rodriguez-Guerra M, Jadhav P, Vittorio TJ. Current treatment in COVID-19 disease: a rapid review. Drugs Context. 2021;10:10-3.

10. Hayakawa M, Takano K, Kayashima M, et al. Management of a COVID-19 Patient during ECMO: paying attention to acquired von willebrand syndrome. J Atheroscler Thromb. 2021;28:396-401.

Publisher's Note Springer Nature remains neutral with regard to jurisdictional claims in published maps and institutional affiliations. 